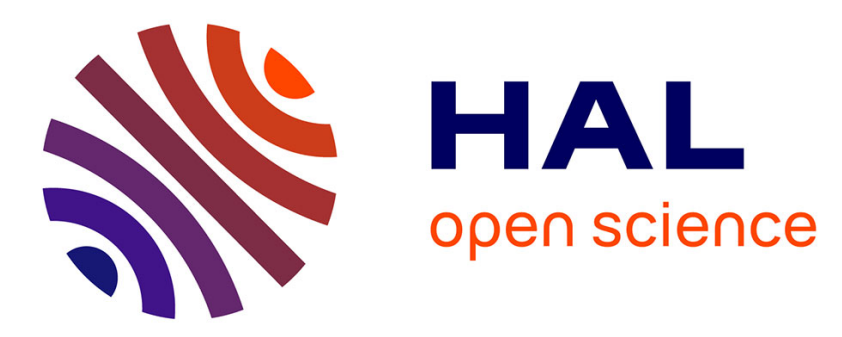

\title{
Fine control of terahertz radiation from filamentation by molecular lensing in air
}

\author{
Magali Durand, Yi Liu, Aurélien Houard, André Mysyrowicz
}

\section{To cite this version:}

Magali Durand, Yi Liu, Aurélien Houard, André Mysyrowicz. Fine control of terahertz radiation from filamentation by molecular lensing in air. Optics Letters, 2010, 35 (10), pp.1710-1712. 10.1364/OL.35.001710 . hal-00487635

\section{HAL Id: hal-00487635 \\ https://hal-polytechnique.archives-ouvertes.fr/hal-00487635}

Submitted on 30 May 2010

HAL is a multi-disciplinary open access archive for the deposit and dissemination of scientific research documents, whether they are published or not. The documents may come from teaching and research institutions in France or abroad, or from public or private research centers.
L'archive ouverte pluridisciplinaire HAL, est destinée au dépôt et à la diffusion de documents scientifiques de niveau recherche, publiés ou non, émanant des établissements d'enseignement et de recherche français ou étrangers, des laboratoires publics ou privés. 


\title{
Fine Control of THz radiation from filamentation by molecular lensing in air

\author{
M. Durand ${ }^{1,2}$, Y. Liu ${ }^{1}$, A. Houard ${ }^{1}$ \& A. Mysyrowicz ${ }^{1}$ \\ ${ }^{1}$ Laboratoire d'Optique Appliquée, ENSTA ParisTech - Ecole Polytechnique, CNRS UMR 7639, \\ Palaiseau, 91761, France \\ ${ }^{2}$ Département d'Optique Théorique et Appliquée, ONERA, 91761 Palaiseau, France
}

\begin{abstract}
We demonstrate a method to control remotely the Terahertz (THz) source in air based on the bifilamentation of femtosecond laser pulses. By fine tuning the time delay between the two pulses, a significant modulation of the $\mathrm{THz}$ intensity from bifilamentation is observed. The phenomenon is attributed to the molecule quantum lensing effect around the air molecule revival time which changes the separation between the two neighboring plasma producing filaments.
\end{abstract}

In the past few years, considerable progress has been achieved in the development of $\mathrm{THz}$ radiation sources and their applications. Many applications in security [1], health [2] or art preservation [3] require $\mathrm{THz}$ illumination of distant targets or samples. This raises a serious problem because of the strong absorption of $\mathrm{THz}$ radiation by water vapor. For instance, a $\mathrm{THz}$ pulse around $1.5 \mathrm{THz}$ is reduced by $12.05 \mathrm{~dB}$ within $50 \mathrm{~m}$ of propagation in air with humidity content of only 39.4\% [4]. Recently, several approaches have addressed this issue.

One approach exploits the femtosecond filamentation process in air. The plasma column formed by a filament emits bursts of $\mathrm{THz}$ radiation along a forward oriented hollow cone [5]. Since it is easy to displace the filament plasma longitudinally, the $\mathrm{THz}$ radiation source can be placed in the immediate proximity of a remote sample. However, this type of $\mathrm{THz}$ source has a low conversion efficiency, on the order of $10^{-9}$, and suffers from its radial polarization pattern along the emission cone which makes it unsuitable in some applications.

The drawbacks of single filamentation $\mathrm{THz}$ source can be corrected in several ways. By applying a transverse DC bias on the plasma column, it is possible to obtain a linearly polarized $\mathrm{THz}$ emission with a three orders of magnitude enhancement and a maximum intensity emitted along the laser propagation axis [6]. However this requires an external electric field at the position of the filament plasma and therefore cannot be considered a remote method stricto sensu. In a different approach, one can use a two-color femtosecond laser pulse, at $\omega$ and $2 \omega$ with sufficient intensity to ionize air [7,8]. Strong enhancement of the $\mathrm{THz}$ radiation with peak field amplitude as high as $400 \mathrm{kV} / \mathrm{cm}$ has been reported with this method using tightly focused laser beams. However, for remote illumination filamentation is unavoidable at high laser powers $P>5 \mathrm{GW}$. A saturation of the $\mathrm{THz}$ intensity occurs because of the dispersion between the $\omega$ and $2 \omega$ components of the bicolor laser field [9].

A last technique used is called bifilamentation; it involves the creation of two closely lying filaments, using a sequence of two single color laser pulses [10]. Bifilamentation radiates up to 60 times more than the transition-Cherenkov $\mathrm{THz}$ radiation from a single filament. Moreover, this enhanced $\mathrm{THz}$ emission is radiated in the forward direction and its polarization can be easily controlled by the orientation of the two filaments. The principle of this enhanced $\mathrm{THz}$ radiation can be qualitatively explained with a transmission line model. After the formation of a plasma column by the first filament, which remains for a few nanoseconds corresponding to the plasma recombination time, the second filament forms its own plasma. Due to the mutual conductance and inductance, these two parallel plasma columns can be viewed as a transmission line sustaining a perpendicular current with respect to the propagation axis. The transverse current oscillating at the characteristic plasma frequency of 
the columns $\sim 0.5-1 \mathrm{THz}$ is at the origin of efficient $\mathrm{THz}$ emission. As the plasma lifetime is of a few nanoseconds, the $\mathrm{THz}$ enhancement appears for corresponding delays between the two pulses. On the other hand, alignment between the filaments is problematic when remote illumination is the objective: $\mathrm{THz}$ enhancement is suppressed if the two filaments are disjoint, or if they overlap completely.

To evaluate the required alignment accuracy, we first studied the influence of the distance $d$ between the two filaments on the enhanced $\mathrm{THz}$ radiation. The laser used was a commercial femtosecond laser system (Alpha100, Thales), which delivers horizontally polarized pulses of $50 \mathrm{fs}$ with up to $15 \mathrm{~mJ}$ energy at a repetition rate of $100 \mathrm{~Hz}$. We used a Mach-Zender interferometer with an incorporated delay line to produce a sequence of two laser pulses. The two pulses were then focused by two $f=100 \mathrm{~cm}$ lenses in order to reduce the distance required for onset of filamentation. A set of mirrors and a three dimensions translation stage supporting one of the lenses, allowed us to precisely align the two filaments and to adjust the horizontal separation $d$ between them. The forward $\mathrm{THz}$ radiation was collected with a Teflon lens and sent to a heterodyne $\mathrm{THz}$ detector, which is sensitive to the horizontally polarized 0.1 $\mathrm{THz}$ component of the $\mathrm{THz}$ radiation with a bandwidth of $4 \mathrm{GHz}$. The detector was $20 \mathrm{~cm}$ away from the end of the filament. All experimental data were averaged over three hundred laser shots.

In Fig. 1, the measured $\mathrm{THz}$ intensity is presented as a function of the filament separation. The delay between the two pulses was set to $1 \mathrm{ps}$. The acceptance angle of the detector was set to $\sim 0.8^{\circ}$ along the filament axis. These results show that there is an optimum distance $d$ between the two filaments for maximum $\mathrm{THz}$ emission, as expected from the bifilamentation transmission line model. This maximum appears when the two plasmas filaments are just about to be separated. This alignment is critical since the separation between the maximum and minimum of THz output is on the order of $100 \mu \mathrm{m}$.

Recently it has been shown that one can deflect a filament at distance by exploiting field-free refractive index revivals $[11,12]$. A short intense optical pulse induces a transient alignment of dipolar molecules along the laser field direction. Quantum mechanically, most of the rotational states which have a component along the laser field will be excited with a fixed phase relationship. Similarly to the mode locking process in laser oscillator, the sum of these rotational modes produces periodic, sharp molecular alignments with a period determined by the constant of rotation of each considered molecule. Due to the different polarizabilities of the molecules with respect to their orientation, these alignments are accompanied by retarded changes of the index of refraction.

Fig. 2 shows the calculated response $R(t)$ of oxygen and nitrogen as a function of time given by the expression, $R(t)=n_{2, \text { rot }} \sum_{j=0}^{\infty} F_{J} \sin \left(-\omega_{J} t\right)$, where $n_{2 r o t}$ is the overall magnitude of the effect given by: $n_{2, \text { rot }}=32 \beta^{2}(\omega) \pi^{2} N / h c n_{0}$, with $\omega_{J}=4 \pi B c(2 J+3)$ the angular frequency difference between the coupled rotational levels, $B$ the rotational constant of the molecule, $N$ the density of the considered gas, $h$ the Planck's constant, $c$ the speed of light, $n_{0}$ the linear refractive index at the laser wavelength and $\beta(\omega)$ the anisotropy of the molecular polarizability [13]. The response of the molecular gas is the coherent sum of the response of each level of rotation. $F_{J}$ corresponds to the contribution of these rotation levels which have been excited by the laser pulse: $F_{J}=\left(\rho_{J+2}-\rho_{J}\right) Z_{J}(J+1)(J+2) /(2 J+3)$, with $\rho_{J}$ the population of the $J$ level and $Z_{J}$ is a coefficient which depends on the molecule considered and on the parity of $J$. In air, this phenomenon is mainly arising from nitrogen and oxygen molecules which have revival times of $8.4 \mathrm{ps}$ and $11.6 \mathrm{ps}$ respectively. 
A second probe pulse will be influenced by the field free change of refractive index and will be attracted or repelled depending on the sign of the change of index $[11,12]$. This suggests that one should be able to control remotely the distance between the two filaments in the bifilamentation process just by finely tuning the delay between the two pulses. This would provide a technique to correct at distance a default in the alignment of the bifilament, or to control precisely the gain of the $\mathrm{THz}$ radiation by bifilamentation.

In Fig. 3 (a), we present the intensity of the $\mathrm{THz}$ signal in air as a function of the delay between the two pulses around the half revival time of $\mathrm{N}_{2}$. As can be seen there is a strong modulation of the $\mathrm{THz}$ around the half revival time of $\mathrm{N}_{2}$ in air. Similar measurements performed in argon are shown in Fig. 3 (b) and no modulation is observed. This confirms that $\mathrm{THz}$ emission can indeed be controlled by the quantum wake effect since the phenomenon appears at the precise time of revival and only in molecular gases. In Fig. 3 (a), we can see that the $\mathrm{THz}$ radiation was not at its maximum before the time of the revival but this maximum could be reached by increasing the delay between the two pulses.

We have performed systematic measurements around half and full revival in pure $\mathrm{N}_{2}$ and $\mathrm{O}_{2}$ (see Fig. 4). For these experiments, the filaments separation was first optimized for maximum $\mathrm{THz}$ emission at a time delay without molecular lensing. As a result, a fine tuning around the revival time can only lead to a decrease of the $\mathrm{THz}$ radiation, since it will either way deviate from the optimum distance. There is an exception at a singular delay time when no net refractive index change occurs and for which the optimal $\mathrm{THz}$ signal is recovered. This is indeed the behavior observed experimentally.

To confirm that the $\mathrm{THz}$ intensity modulation is due to the molecule lensing effect, we have performed damage pattern experiments with glass plates. The damages allow monitoring the distance between the two filaments. Fig. 5 presents damage patterns obtained in nitrogen at three characteristic time delays specified in Fig. 4 (c). The damage in Fig. 5 (a) was taken at time delay away from the molecule alignment revival $\left(\tau_{d}=8.15 \mathrm{ps}\right)$, displaying two neighboring filaments with an optimal separation. At time delay $\tau_{d}=8.32 \mathrm{ps}$, when the $\mathrm{THz}$ intensity shows a minimum (position 2), the damage pattern in Fig. 5 (b) clearly indicates a fusion of the two filaments due to positive molecule lensing effect. In the middle of the $\mathrm{N}_{2}$ molecule revival ( $\tau_{d}=8.42 \mathrm{ps}$ ), the local refractive index comes back to its initial value and a maximum $\mathrm{THz}$ intensity is recovered (see Fig. 4 (c)).

To conclude we have demonstrated the influence of quantized molecule rotation states on the $\mathrm{THz}$ radiation created by two copropagating filaments. A fine tuning of the delay between two laser pulses provides an effective remote technique for optimization of the distance between two plasma channels created by filamentation and thereby of the $\mathrm{THz}$ intensity. An extension to multifilament patterns should be possible. 
References:

[1] M. K. Choi, A. Bettermann and D. W. Van der Weide, "Potential for detection of explosive and biological hazards with electronics terahertz systems," Phil. trans. R. Soc. Lond. A, 362, 337-349 (2004)

[2] E. Pickwell and V. P. Wallace, "Biomedical applications of terahertz technology," J. Phys. D: Appl. Phys. 39, R301-R310 (2006)

[3] J. B. Jackson, M. Mourou, J. F. Whitaker, I. N. Duling, S. L. Williamson, M. Menu, G. A. Mourou, "Terahertz imaging for non-destructive evaluation of mural paintings," Opt. Commun. 281, 527-532 (2008)

[4] K. J. Linden and W. R. Neal, "Terahertz laser based standoff imaging system," in Proceedings of the 34th Applied Imagery and Pattern Recognition Workshop (IEEE, 2005)

[5] C. D'Amico, A. Houard, M. Franco, B. Prade, and A. Mysyrowicz, "Conical Forward THz Emission from Femtosecond-Laser-Beam Filamentation in Air," Phys. Rev. Lett. 98, 235002 (2007)

[6] A. Houard, Y. Liu, B. Prade, V. T. Tikhonchuk, and A. Mysyrowicz, "Strong Enhancement of Terahertz Radiation from Laser Filaments in Air by a Static Electric Field," Phys. Rev. Lett. 100, 255006 (2008)

[7] X. Xie, J. Dai, and X.-C. Zhang, "Coherent Control of the THz Wave Generation in Ambient Air," Phys. Rev. Lett. 96, 075005 (2006)

[8] T. Bartel, P. Gaal, K. Reimann, M. Woerner, and T. Elsaesser, "Generation of single-cycle THz transients with high electric-field amplitudes," Opt. Lett. 30, 2805-2807 (2005)

[9] Y. Liu, A. Houard, M. Durand, B. Prade, and A. Mysyrowicz, "Maker fringes in the Terahertz radiation produced by a 2-color laser field in air," Opt. Express 17, 11480-11485 (2009)

[10] Y. Liu, A. Houard, B. Prade, S. Akturk, and A. Mysyrowicz, "Terahertz radiation source in air based on bifilamentation of femtosecond laser pulses," Phys. Rev. Lett. 99, 135002 (2007)

[11] S. Varma, Y.-H. Chen, and H. M. Milchberg, "Trapping and destruction of long-range high-Intensity optical filaments by molecular quantum wakes in air," Phys. Rev. Lett. 101, $205001(2008)$

[12] F. Calegari, C. Vozzi, S. Gasilov, E. Benedetti, G. Sansone, M. Nisoli, S. De Silvestri, and S. Stagira, "Rotational Raman Effects in the wake of Optical Filamentation," Phys. Rev. Lett. 100, 123006 (2008)

[13] J.-F. Ripoche, G. Grillon, B. Prade, M. Franco, E. Nibbering, R. Lange and A. Mysyrowicz, "Determination of the time dependence of $n 2$ in air," Opt. Commun. 135, 310315 (1997) 


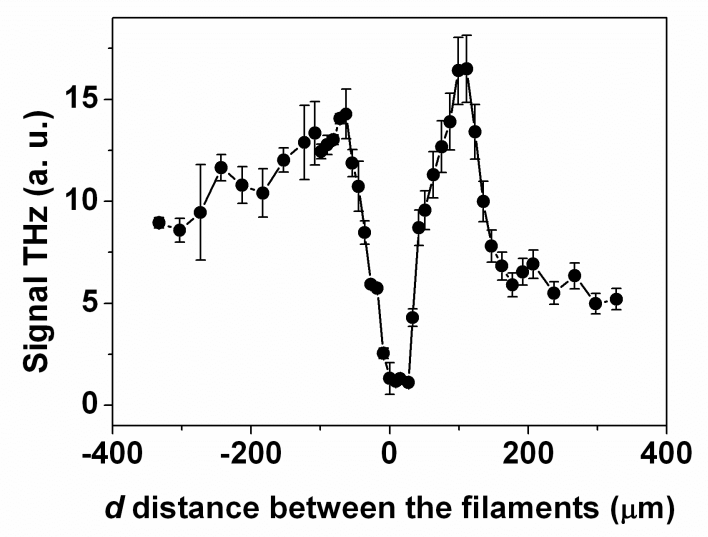

Fig. 1. THz intensity as a function of the distance between the two filaments. The two filaments were created by two laser pulses of $50 \mathrm{fs}$ duration and $1.5 \mathrm{~mJ}$ energy. The maximum enhancement of the $\mathrm{THz}$ radiation is on the order of 30 as compared to a single filament. .

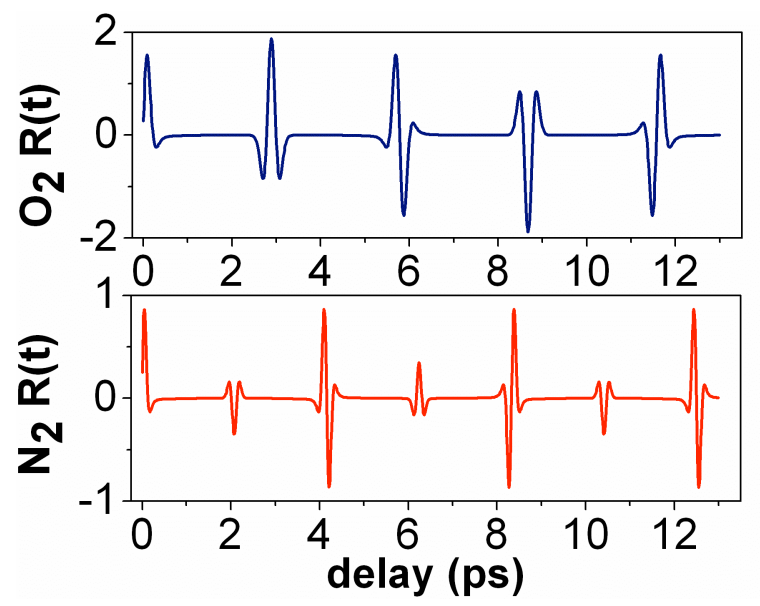

Fig. 2. Calculated response of the $\mathrm{O}_{2}$ (top) and $\mathrm{N}_{2}$ (bottom) molecules.

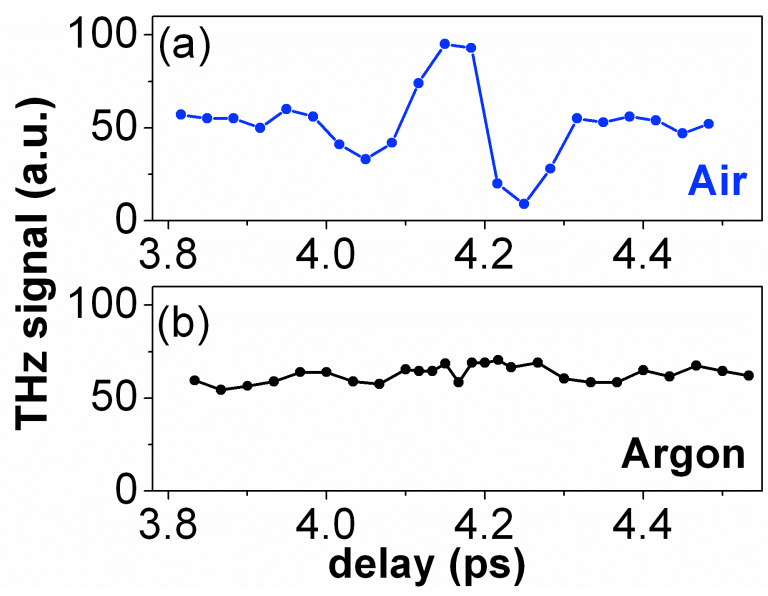

Fig. 3. THz intensity produced by bifilamentation as a function of the delay between the two laser pulses (pulse energy: $2 \mathrm{~mJ}$; pulse duration: $50 \mathrm{fs}$ ) around the half-revival period of $\mathrm{N}_{2}$ molecule in air (a) and in Argon (b). The enhancement factor of the THz radiation in case (a) is about 10 . 

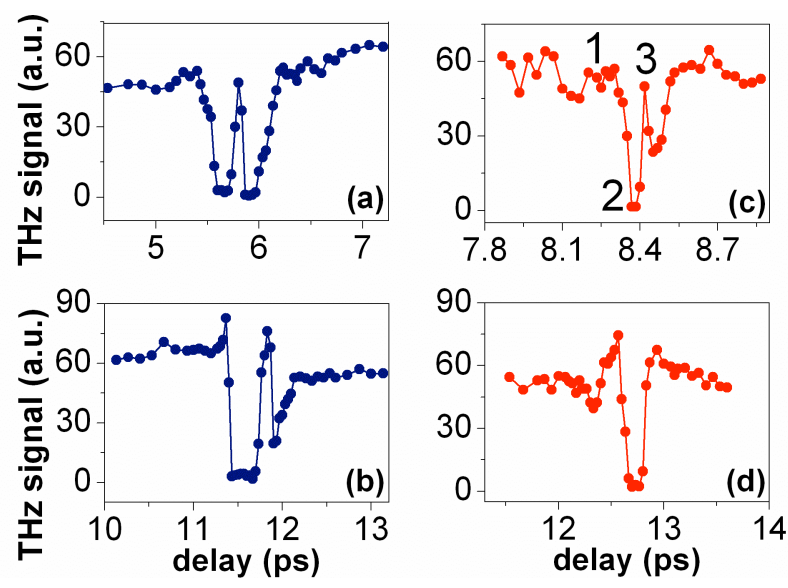

Fig. 4. (a) and (b), measurement of the variation of the $\mathrm{THz}$ intensity as a function of delay between the two laser pulses around the half and full revival period of $\mathrm{O}_{2}$. (c) and (d) show the same measurements in $\mathrm{N}_{2}$ for time around 1 and $3 / 2$ of the revival period. Enhancement of the $\mathrm{THz}$ radiation is about 20 for each figure.
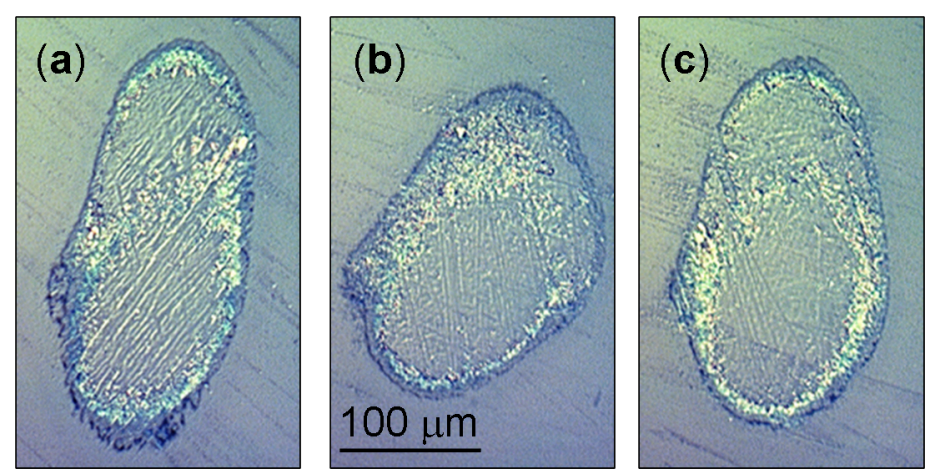

Fig. 5. (a), (b) and (c), damage patterns produced by the bifilament on a glass plate sample in $\mathrm{N}_{2}$ at the three sequential time delays indicated in Fig. 4 (c). 